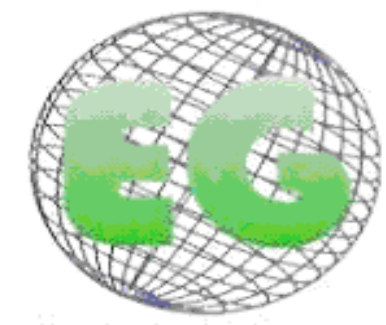

ISSN 1695-6141

$N^{\circ} 26$

\title{
Relación entre los resultados del Electroencefalograma digital y la evaluación clínica, neuropsicológica e imagenológica en pacientes con diagnóstico clínico de Parálisis Cerebral
}

Relation between digital Electroencephalogram clinic results, neuropsychological and imagenologic evaluation in patients with cerebral palsy clinic diagnostic

\section{*Zaldívar Bermúdez, M., " ${ }^{* *}$ Morales Chacón, L.,"**Sánchez Curuneaux, A., ****Trápaga Quincoses, O., *****Vera Cuesta, H., ***** García,ME.}

*Técnico en Neurofisiología clínica. E-mail marilyn@neuro.ciren.cu **Doctora en Ciencias. Jefa del Servicio de Neurofisiología clínica ***Enfermero General. Técnico en Neurofisiología clínica. ${ }^{* * * * E s p e c i a l i s t a ~ e n ~}$ Neurofisiología clínica y Máster en Neurociencias. ${ }^{* * * * * E s p e c i a l i s t a ~ e n ~ N e u r o l o g i ́ a ~ i n f a n t i l ~ y ~ M a ́ s t e r ~ e n ~}$ Biogenética. ${ }^{* * * * * *}$ Especialista en Neuropsicología y Máster en Neurociencias. Centro Internacional de Restauración Neurológica (CIREN). La Habana. Cuba.

Palabras clave: parálisis cerebral; electroencefalograma; epilepsia; retraso mental; retardo del desarrollo psicomotor; resonancia magnética nuclear (RMN)

Keywords: cerebral palsy; electroencephalogram; epilepsy; mental delay; phychomotor retardation; magnetic resonance image (MRI)

\section{RESUMEN}

Objetivo: Evaluar la relación existente entre los resultados del Electroencefalograma (EEG) digital y las anomalías clínicas, neuropsicológicas e imagenológicas detectadas en pacientes con el diagnóstico clínico de Parálisis Cerebral (PC).

Sujetos y Métodos: Se realizó un estudio descriptivo longitudinal retrospectivo de los informes de EEG emitidos por un especialista en Neurofisiología clínica en 64 pacientes con el diagnóstico clínico de PC. Los datos fueron registrados desde enero del año 2000 hasta diciembre del año 2005 en el Laboratorio de Neurofisiología Clínica del CIREN. Incluimos a pacientes con edades entre 2 y 15 años, con diagnóstico revisado de la historia clínica de PC. Se tabularon las variables evaluadas en los registros de EEG, y se relacionaron con variables clínicas, neuropsicológicas e imagenológicas. Todos los registros se realizaron en un Equipo MEDICID 4 Neuronic SA de producción cubana con el software Track Walker 2.

Resultados: Se obtuvo que el $79 \%$ del los registros electroencefalográficos fueron patológicos. Predominó la actividad epileptiforme en un 58\%. El tipo de PC en nuestra casuística fue la espástica para un $50 \%$. La causa de la PC fue perinatal en un $43 \%$. Las enfermedades asociadas fueron Retardo del desarrollo psicomotor 
(RDP), Retraso mental (RM) y epilepsia. Se evidenció una asociación estadísticamente significativa entre la presencia de actividad epileptiforme y la epilepsia, entre los resultados del EEG y la presencia de lesión bilateral, así como entre la actividad epileptiforme y la presencia de RM, RDP y epilepsia.

Conclusiones: Se constata una asociación estadísticamente significativa entre las anomalías epileptiformes y el diagnóstico clínico de epilepsia en pacientes con PC. Las anomalías del EEG se asocian mayormente con la extensión bilateral de la afectación motora, y con las lesiones detectadas mediante los estudios de Resonancia Magnética Nuclear (RMN). Existe una tendencia a la asociación entre las alteraciones Electroencefalográficas, el tipo de PC y la presencia de retardo en el desarrollo psicomotor y retraso mental. El EEG es un medio diagnóstico útil en la evaluación funcional de los pacientes con PC.

\section{ABSTRACT}

Objetive: To evaluate the relation between digital Electroencephalogram (EEG) results and clinic, neuropsychological and imagenologic anomaly detected in patients with Cerebral Palsy (CP) diagnosis.

Subject and method: Descriptive longitudinal retrospective study was made of EEG informs emitted by a clinical neurophysiologic specialist in 64 patients with CP diagnosis. The information was recorded from January 2000 to December 2005 in a Clinical Neurophysiologist laboratory of the International Center of Neurological Restoration. It included patients between 2 and 15 years old, with CP diagnostic checked in clinical history. The variables assessed were tabulated in EEG records and were associated to clinical, neuropsychology and imagenologic variables. All recordings were performed with the MEDICID 4 Neuronic SA equipment made in Cuba with Track Walker 2 software.

Results: $79 \%$ of the electroencephalographic records were pathological. In $58 \%$ of the cases there was a predominance of epileptiform activity. $50 \%$ suffered from spastic CP. The cause of CP was perinatal in $43 \%$. The other associated conditions were Phychomotor Retardation (PR), Mental Delay (MD) and epilepsy. There was a statistically significant association between the presence of epileptiform activity and epilepsy between the EEG records and bilateral lesion and between the epileptiform activity and the presence of MD, PR and epilepsy.

Conclusions: Our results demonstrate the exististence of a statistically significant association between epileptiform anomaly and epilepsy clinical diagnosis. It was confirmed that EEG anomaly is associated with bilateral extension of motor affectation and lesions detected with Magnetic Resonance Image (MRI) studies. There was an association tendency between electroencephalographic alteration, CP type, PR and MD presence. EEG is a useful diagnostic way for the functional evaluation of CP patients.

\section{INTRODUCCIÓN}

El electroencefalograma (EEG) es uno de los pocos métodos de que disponemos para evaluar la función del sistema nervioso central. Además de tener las ventajas de ser no cruento y de bajo costo. Las modernas técnicas de neuroimágenes, como la Tomografía Axial Computarizada (TAC) y Resonancia Magnética Nuclear (RMN) son métodos estructurales que nos permiten ver la lesión pero no nos informan acerca del proceso fisiopatológico que se sucede en la misma ${ }^{(1)}$

La parálisis cerebral (PC) es un término diagnóstico que se utiliza para describir un grupo de síndromes motores secundarios a alteraciones precoces del desarrollo cerebral. La PC puede tener un origen malformativo, genético, metabólico, isquémico, infeccioso u otras causas adquiridas que producen un fenotipo neurológico común. ${ }^{(2)}$

La PC es la forma más frecuente y costosa de discapacidad crónica motora de inicio en la infancia, con una prevalencia de 2/1.000. ${ }^{(2,3)}$

La incidencia global de PC se sitúa alrededor de un $2 \%$ de los niños nacidos vivos, aunque esta cifra está basada en estadísticas de otros países y varía en las diferentes series, en función de los criterios de selección y tipo de comunidad estudiada. La clasificación 
fisiológica identifica la principal anomalía motora, mientras que la taxonomía topográfica indica las extremidades afectadas. ${ }^{(2)}$

La PC también se asocia con frecuencia a una serie de discapacidades del desarrollo, incluidos retraso mental (RM), epilepsia y anomalías visuales, auditivas, del habla, cognitivas y conductuales. La discapacidad motora puede ser el menor de los problemas del niño: Hemiplejia espástica, Diplejía espástica, Tetraplejía espástica, la PC atetoide. ${ }^{(2)}$

El niño con el diagnóstico de PC desde su nacimiento, y más tarde de adolescente y adulto, va a necesitar de más atenciones y cuidados especiales. Un equipo interdisciplinar de profesionales: médicos, fisioterapeutas, terapeutas ocupacionales, logopedas, psicólogos etc., coordinados por el médico rehabilitador, y en contacto muy directo con los padres, debería facilitar la atención más adecuada para intentar paliar y minimizar las secuelas que se derivan de la lesión cerebral. Facilitamos, de este modo, el desarrollo integral del paralítico cerebral. ${ }^{(4)}$

En este trabajo nos proponemos evaluar la relación existente entre los resultados del EEG digital y las anomalías clínicas, neuropsicológicas e imagenológicas detectadas en pacientes con Parálisis cerebral infantil.

\section{OBJETIVO}

Evaluar la relación existente entre los resultados del Electroencefalograma (EEG) digital y las anomalías clínicas, neuropsicológicas e imagenológicas detectadas en pacientes con el diagnóstico clínico de Parálisis Cerebral.

\section{SUJETOS Y MÉTODOS}

Se realizó un estudio descriptivo longitudinal retrospectivo de los informes de EEG emitidos por un especialista en Neurofisiología clínica en 64 pacientes con el diagnóstico clínico de PC infantil.

Los datos fueron registrados desde enero del año 2000 hasta diciembre del año 2005 en el Laboratorio de Neurofisiología Clínica del Centro Internacional de Restauración Neurológica. Incluimos pacientes con edades entre 2 y 15 años, con diagnóstico revisado de la historia clínica de PC.

Se tabularon las variables evaluadas en los registros de EEG, y se relacionaron con variables clínicas (presencia de crisis y tipo de PC), neuropsicológicas (RM y RDP e imagenológicas) presencia y localización de anomalía estructural en RMN.

Todos los registros se realizaron en un Equipo MEDICID 4 Neuronic SA de producción cubana con el software Track Walker 2.

Para evaluar la relación entre las variables Electroencefalográficas y las clínicas, imagenológicas y neuropsicológicas (cualitativas) utilizamos el programa STATISTIC 6. Se realizó un test exacto de Fisher y se consideraron significativas las relaciones con $\mathrm{p}<0.05$.

\section{RESULTADOS}

El 79\% de los registros electroencefalográficos fueron patológicos. En los registros de EEG se analizó la existencia de alteración en la actividad de base y la presencia de actividad 
epileptiforme. En nuestra casuística predominó la presencia de actividad epileptiforme lo cual representa un $58 \%$, el trastorno en la actividad de base fue en un $22 \%$ y fueron normales en un $20 \%$.

El tipo de PC sobresaliente en nuestra casuística fue la espástica para un 50 \% (fig.1).

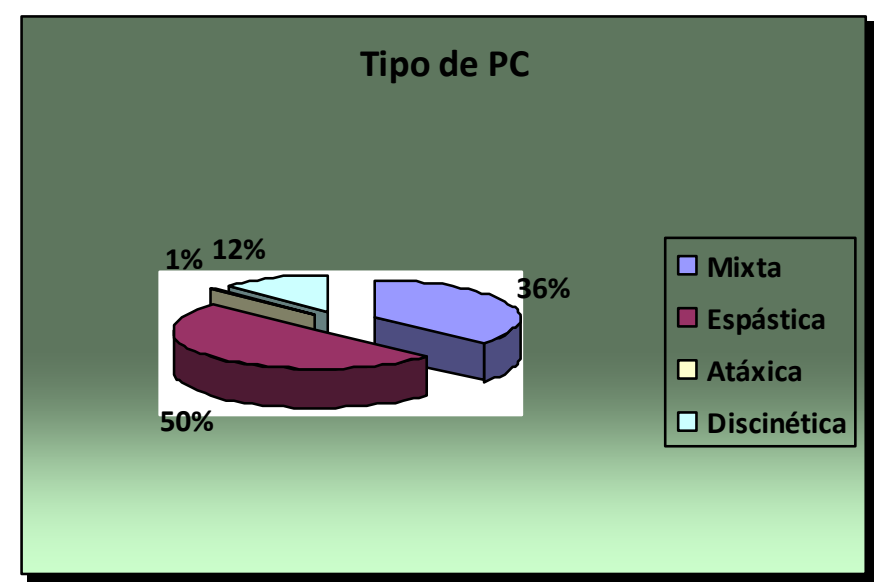

Fig. 1. Porciento de pacientes según el tipo de PC.

Según la extensión de la PC la cuadriparesia fue la predominante para un 58 \% (fig.2). La causa de la PC mayormente fue perinatal para un $43 \%$ y dentro de las enfermedades asociadas encontramos RDP, RM y la epilepsia.

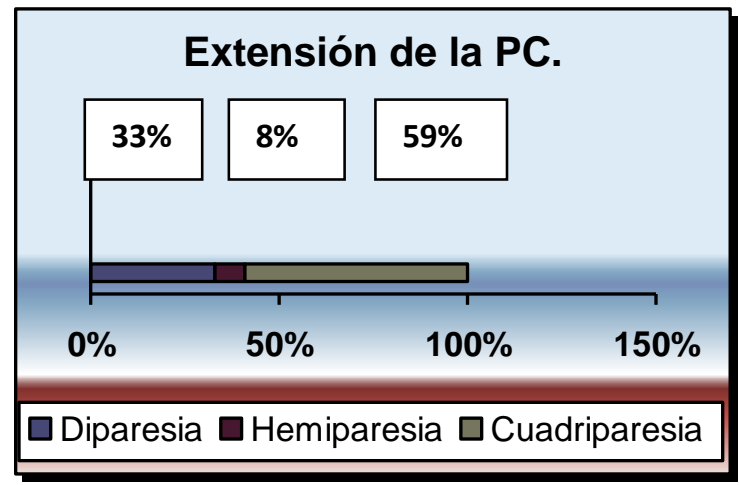

Fig.2. Porciento de pacientes según la extensión de la PC.

Particularizando en la actividad epileptiforme podemos señalar que 37 pacientes del total presentaban actividad epileptiforme en los registros de EEG lo cual representa un 58\%, y de estos, 27 pacientes presentaban como enfermedad asociada la epilepsia lo que constituye un $42 \%$, existiendo por tanto una asociación estadísticamente significativa entre estas dos variables $(p=, 0014)$ como se muestra en la tabla 1 .

Con relación a la asociación existente entre los resultados del EEG y la extensión de la lesión de la PC, podemos señalar que 59 pacientes del total presentaban lesión bilateral para un $92 \%$, de estos 47 presentaban el EEG patológico para un $73 \%$, existiendo una asociación significativa entre estas variables $(p=0,000)$. 
Tabla 1. - Relación entre la actividad epileptiforme y el diagnóstico de epilepsia.

\begin{tabular}{|c|c|c|c|}
\hline \multirow{2}{*}{ Epilepsia } & \multicolumn{2}{|c|}{ Actividad Epileptiforme } & \multirow{2}{*}{ Total } \\
\cline { 2 - 3 } & Sí & No & \\
\hline Sí & $27(42 \%)$ & $8(12 \%)$ & $35(55 \%)$ \\
\hline No & $10(16 \%)$ & $19(30 \%)$ & $29(45 \%)$ \\
\hline Total & $37(58 \%)$ & $27(42 \%)$ & $64(100 \%)$ \\
\hline Chi ${ }^{2}=10,15$ & $\mathrm{p}=, 0014$ \\
\hline
\end{tabular}

En cuanto a la localización de la actividad epileptiforme predominaron en los registros electroencefalográficos las anomalías no multifocales $(32,8 \%)$ como se muestra en la tabla 2. Se evidenció una asociación estadísticamente significativa entre esta anomalía y la ocurrencia de RM, RDP y epilepsia.

Adicionalmente evidenciamos una asociación entre resultados del EEG y las imágenes estructurales donde 49 pacientes del total tenían alteraciones en los estudios de imágenes lo cual representa un $76 \%$, notándose mayormente que 37 de estos presentaban alteración en el EEG para un $57,8 \%$.

Tabla 2.- Relación entre la presencia de Actividad Epileptiforme Multifocal y el diagnóstico de Epilepsia, Retardo en el desarrollo psicomotor (RDP) y Retraso Mental (RM).

\begin{tabular}{|c|c|c|c|c|c|c|c|}
\hline \multirow{2}{*}{\multicolumn{2}{|c|}{$\begin{array}{l}\text { Prestencia te } \\
\text { AE } \\
\text { Multifocal } \\
\text { en el EEG }\end{array}$}} & \multicolumn{2}{|c|}{ Retraso Mental } & \multicolumn{2}{|c|}{ Epilepsia } & \multicolumn{2}{|c|}{ RDP } \\
\hline & & SI & NO & SI & NO & SI & NO \\
\hline \multirow{2}{*}{$\begin{array}{c}\text { MULT } \\
\text { IFOC } \\
\text { AL }\end{array}$} & & $5(7,8 \%)$ & $7(10,9 \%)$ & $12(19 \%)$ & $0(0 \%)$ & $7(10,9 \%)$ & $5(7,8 \%)$ \\
\hline & No & $\begin{array}{l}21(32,8 \\
\%)\end{array}$ & $31(48,4 \%)$ & $23(36 \%)$ & $29(45,3 \%)$ & $23(35,9 \%)$ & $29(45,3 \%)$ \\
\hline \multicolumn{2}{|c|}{$\begin{array}{c}\text { Total } \\
\text { parcial }\end{array}$} & $\begin{array}{l}26(40,6 \\
\%)\end{array}$ & $38(59,4 \%)$ & $35(55 \%)$ & $29(45,3 \%)$ & $30(46,9 \%)$ & $34(53,1 \%)$ \\
\hline \multicolumn{2}{|c|}{ Total } & \multicolumn{2}{|c|}{$64(100 \%)$} & \multicolumn{2}{|c|}{$64(100 \%)$} & \multicolumn{2}{|c|}{$64(100 \%)$} \\
\hline \multicolumn{2}{|c|}{$\begin{array}{l}\text { Asociación } \\
\text { estadística }\end{array}$} & \multicolumn{2}{|c|}{$\begin{array}{l}\mathrm{Chi}^{2}=6,04 \\
\mathrm{p}=0,0140\end{array}$} & \multicolumn{2}{|c|}{$\begin{aligned} \mathrm{Chi}^{2} & =10,82 \\
\mathrm{P} & =, 0010\end{aligned}$} & \multicolumn{2}{|c|}{$\begin{array}{c}\mathrm{Chi}^{2}=10,32 \\
\mathrm{p}=0,0013\end{array}$} \\
\hline
\end{tabular}

\section{DISCUSIÓN}

En nuestro trabajo se evidenció una asociación entre las anomalías del EEG, la extensión de la PC, la ocurrencia de crisis epilépticas, el RDP, el RM y las anomalías estructurales evidenciadas por RMN. 
Utilizamos la clasificación de la PC referida en la literatura según el trastorno motor predominante y la extensión de la lesión, lo cual es de utilidad para la orientación del tipo de tratamiento así como para el pronóstico evolutivo. ${ }^{(5)}$

A Faoro ${ }^{(1)}$ y Poó ${ }^{(5)}$ plantean que el EEG no brinda información para el diagnóstico de PC, pero dado que un porcentaje significativo de niños con PC desarrollan epilepsia, se hace necesaria su práctica para la detección de los pacientes con más riesgo y, por supuesto, para el seguimiento de aquellos que tienen crisis epilépticas. En nuestro estudio el $55 \%$ presentaban crisis epilépticas y el $58 \%$ actividad epileptiforme, lo cual plantea la necesidad de realizar EEG en estos pacientes.

Maragoto y cols plantean que en su muestra de los estudios neurofisiológicos realizados a pacientes con PC, los potenciales somatosensoriales resultaron los que con más frecuencia se encuentran alteraciones con $62.1 \%$, seguido del EEG con $56.3 \%$. ${ }^{(6)}$

Kulak W en el 2003 planteó que más del 50\% de los niños con esta afectación presentan crisis epilépticas de tipo parcial con generalización secundaria y frecuentes estados de mal epiléptico ${ }^{(7)}$. Por su parte Pilar Póo Arguelles en $1995^{(8)}$ y en el $2008^{(5)}$ planteó que aproximadamente la mitad de los niños con PC tienen epilepsia, muy frecuente en pacientes con tetraplejia $(70 \%)$ y riesgo inferior al $20 \%$ en dipléjicos, nuestros resultados resultan congruentes con los expuestos por Kulak W y Poó, pues el $50 \%$ de nuestros pacientes tenían una PC espástica, predominando la cuadriparesia.

Se ha afirmado que la PC ${ }^{(9)}$ y el retraso mental se asocian en un $50 \% .{ }^{(10)}$ El rendimiento cognitivo según datos publicados en el 2008 por Poó ${ }^{(5)}$ desde la normalidad, en un 50-70\% de los casos a un retraso mental severo, frecuentemente en los niños con tetraplejia. El menor grado de retraso lo presentan los niños con diplejía y los hemipléjicos. Problemas de comunicación y de lenguaje, son más frecuentes la PC discinética ${ }^{(5)}$. Nuestros resultados evidencian que 30 del total de los pacientes presentaban como enfermedad asociada el retardo en el desarrollo psicomotor para un 46,9\%, además 26 del total de pacientes presentaron retraso mental para un $40,6 \%$.

Un $43 \%$ de nuestros pacientes la PC fue de causa perinatal. En la literatura se reporta que los factores perinatales pudieran ser: prematuridad, bajo peso, fiebre materna durante el parto, infección Sistema Nervioso Central (SNC) o sistémica, hipoglucemia mantenida, hiperbilirrubinemia, hemorragia intracraneal, encefalopatía hipóxico-isquémica, traumatismo, cirugía cardíaca, etc. ${ }^{(5)}$

En nuestra muestra encontramos una asociación estadísticamente significativa entre los pacientes que presentaban alteraciones epileptiformes en el EEG y las alteraciones en los estudios imagenológicos $(57,8 \%)$. En relación a esto en la literatura se reporta que en ocasiones los hallazgos de la neuroimagen servirán para confirmar la existencia, localización y extensión de la lesión, e incluso de la etiología, aunque no siempre existe relación entre el grado de lesión visible en neuroimagen y el pronóstico funcional. ${ }^{(11)}$

\section{CONCLUSIONES}

1. Se constata una asociación estadísticamente significativa entre las anomalías epileptiformes y el diagnóstico clínico de epilepsia en pacientes con PC. 
2. Las anomalías del EEG se asocian mayormente con la extensión bilateral de la afectación motora, y con las lesiones detectadas mediante los estudios de imágenes estructurales.

3. Existe una tendencia a la asociación entre las alteraciones Electroencefalográficas, el tipo de PC y la presencia de retardo en el desarrollo psicomotor y retraso mental.

4. El EEG es un medio diagnóstico útil en la evaluación funcional de los pacientes con PC.

\section{BIBLIOGRAFÍA}

1.- A Faoro M, F Pereira y M Colmenares. Electroencefalografía neonatal: Su importancia en el proceso de la evolución neurológica de recién nacidos prematuros y a término de alto riesgo. Revista de la facultad de medicina ISNN 0798-469 versión impresa. RFM v.24 n.1 Caracas mar.2001.

2.- Lorente I, Bugie C. Parálisis Cerebral. En: Fegerman N, Fernández E. Buenos Aires: El Ateneo; 1988: 4.1-4.67.

3.- Toledo-González M. Concepto. Etología. Alto riesgo del recién nacido. In Toledo González M, ed. Parálisis cerebral. Madrid. Departamento de estudios y publicaciones del SEREM, 1977.p.25-45.

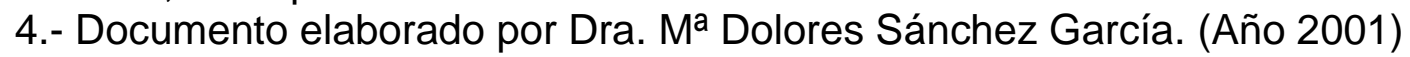

Descargado de www.ortoinfo.com, el primer portal en español especializado en ortopedia. Consultado el 7 de diciembre del 2011.

5.- Pilar Poó Arguelles. Parálisis Cerebral Infantil. Servicio de Neurología. Hospital Sant Joan de Déu, Barcelona. Protocolos Diagnósticos Terapéuticos de la AEP: Neurología Pediátrica. 2008.

6.- Maragoto RC, Vera CH, Cepero MG, Fernández MI, Marín HT. Variabilidad de la eficacia de un programa de restauración neurológica en pacientes con parálisis cerebral. Rev Mex neuroci 2007; 8(2): 119-126.

7.- Kulak W, Sobaniec W. Quantitative EEG analysis in children with hemiparetic cerebral palsy. Neurorehabilitation 2005; 20(2): 75-84) y (Singhi P, Jagirdar S, Khandelwal N, Malhi P. Epilepsy in children with cerebral palsy. J Child Neurol 2003; 18(3): 174-9.

8.- Poó P, Maito Lima J, Sanmartí F. Epilepsia en niños con Parálisis Cerebral. Act Ped Esp 1995; 53:304-308).

9. - Matthews DJ, Wilson P. Cerebral Palsy. In Molnar gabriella E, Alexander Michael A, eds. Pedriatic rehabilitation. Philadelphia. Hanley \& Belfus; 1999. P. 193-217.

10. - Braun K, Satten G, Williams S, Boyle C. Rates and factors associated with the recurrence of mental retardation, cerebral palsy, hearing impairment and vision impairment. Metropolitan Atlanta Developmental Disabilities Surveillance Program 1991-1994. Paedriatr Perinat Epidemiol 2001; 15:5.

11.- Wojciech K, Wojciech S, Bozena K et al. Spastic Cerebral palsy:clinical / Magnetic Resonance Imaging correlation of 129 children. J Children Neurol 2007; 22:8-14.

ISSN 1695-6141

() COPYRIGHT Servicio de Publicaciones - Universidad de Murcia 